\title{
A Theory of Cartesian Product and Factorization of Circulant Graphs
}

\author{
V. Vilfred \\ Department of Mathematics, St. Jude's College, Thoothoor, Tamil Nadu 629 176, India \\ Correspondence should be addressed to V. Vilfred; vilfredkamal@hotmail.com
}

Received 27 May 2013; Revised 14 September 2013; Accepted 16 September 2013

Academic Editor: Wai Chee Shiu

Copyright (C) 2013 V. Vilfred. This is an open access article distributed under the Creative Commons Attribution License, which permits unrestricted use, distribution, and reproduction in any medium, provided the original work is properly cited.

We determine when the Cartesian product of two circulant graphs is also a circulant graph. This leads to a theory of factorization of circulant graphs.

\section{Introduction}

Just as integers can be factored into prime numbers, there are many results on decomposition of structures throughout mathematics [1]. The standard products-Cartesian, lexicographic, tensor, and strong-all belong to a class of products introduced by Imrich and Izbicki [2] and called B-products [3]. Properties of circulant graphs are extensively studied by many authors [2-19] and products of graphs have been studied for almost 50 years now. Sabidussi [20] proved that every (nonnull) graph is the unique product of prime graphs. Broere and Hattingh [3] established that the lexicographic product of two circulant graphs is again circulant. They and Sanders and George [12] established that this is not the case with other products. Alspach and Parsons [5] introduced metacirculant graphs as a generalization of circulant graphs and characterized metacirculant graphs in terms of their automorphism groups. Sanders [11] established that any $B$ product of two circulant graphs is always a metacirculant graph with parameters that are easily described in terms of the product graphs and also established that any metacirculant graph with the appropriate structure is isomorphic to the $B$-product of a pair of circulant graphs. After a graph is identified as a circulant graph, its properties can be derived easily. This paper gives a detailed study of Cartesian product and factorization of circulant graphs similar to the theory of product and factorization of natural numbers. For more details on circulant graphs, see $[9,10]$.

Let $n$ be a positive integer and let $R$ be a subset of $\{1,2, \ldots,[n / 2]\}$. The circulant graph $C_{n}(R)$ has vertices $v_{1}, v_{2}, \ldots, v_{n}=v_{0}$ with $v_{i}$ adjacent to $v_{i+r}$ for each $r \in R$, subscript addition taken modulo $n$. When discussing circulant graphs, we will often assume, without further comment, that the vertices are the corners of a regular $n$-gon, labeled clockwise. Circulant graphs $C_{7}(1,3)$ and $C_{8}(2,4)$ are shown in Figures 1(a) and 1(b).

When $n / 2 \in R$, edge $v_{i} v_{i+n / 2}$ is taken as a single edge while considering the degree of a vertex, but as a double edge while counting number of edges or cycles in $C_{n}(R)[3,6-$ $10,13,14,17,18,21]$. We generally write $C_{n}$ for $C_{n}(1)$ and $C_{n}()$ for $C_{n}(\Phi)=K_{n}^{c}$, the null graph on $n$ vertices. Note that if a graph $G$ is circulant, then its adjacency matrix $A(G)$ is circulant. It follows that if the first row of the adjacency matrix of a circulant graph is $\left[a_{1}, a_{2}, \ldots, a_{n}\right]$, then $a_{1}=0$ and $a_{i}=a_{n-i+2}, 2 \leq i \leq n$.

Throughout this paper, for a set $R=\left\{r_{1}, r_{2}, \ldots, r_{k}\right\}, C_{n}(R)$ denotes $C_{n}\left(r_{1}, r_{2}, \ldots, r_{k}\right)$ where $1 \leq r_{1}<r_{2}<\cdots<r_{k} \leq$ $[n / 2]$; we consider only connected circulant graphs of finite order greater than 2 and all cycles have length at least 3 , unless otherwise specified.

Let $n, r$ be positive integers, $n \geq 2$ and $r<n / 2$. Then, $C_{n}(r)$ consists of a collection of disjoint cycles, $v_{0} v_{r} v_{2 r}, \ldots, v_{0} ; v_{1} v_{1+r} v_{1+2 r}, \ldots, v_{1} ; \ldots ; v_{r-1} v_{2 r-1} v_{3 r-1}, \ldots, v_{r-1}$. If $d=\operatorname{gcd}(n, r)$, then there are $d$ such disjoint cycles and each has length $n / d$. We say that each of these cycles has period $r$, length $n / d$, and rotation $r / d$.

If $r=n / 2$, then obviously $C_{n}(r)$ is just a 1 -factor. Since $C_{n}(R)$ is just the union of the cycles of $C_{n}(r)$ for $r \in R$, we have a decomposition of $C_{n}(R)$. 


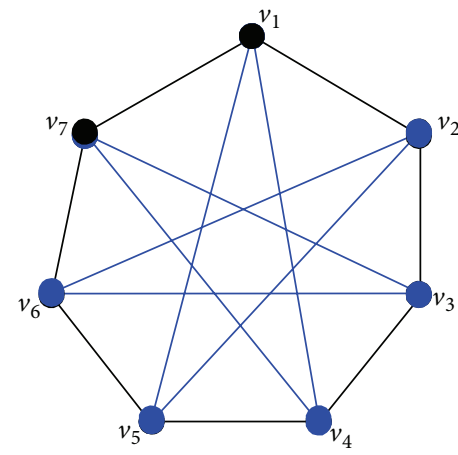

(a)

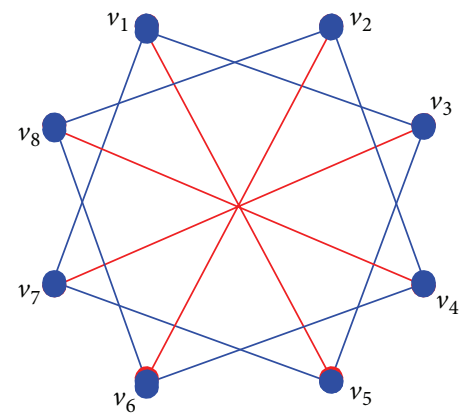

(b)

Figure 1: (a) $C_{7}(1,3)$. (b) $C_{8}(2,4)$.

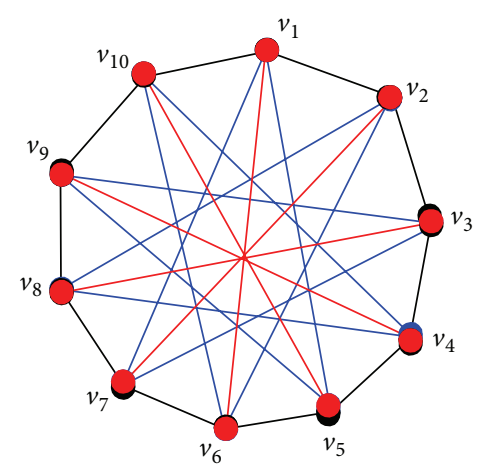

Figure 2: $C_{10}(1,4,5)$.

Theorem 1 (see [7]). Circulant graph $C_{n}(R)$ for a set $R=\left\{r_{1}\right.$, $\left.r_{2}, \ldots, r_{k}\right\}$ is connected if and only if $g c d\left(n, r_{1}, r_{2}, \ldots, r_{k}\right)=1$.

Theorem 2 (see $[15,18])$. Let $r \in R$. Then, in $C_{n}(R)$, the length of a cycle of period $r$ is $n / g c d(n, r)$ and the number of vertexdisjoint periodic cycles of period $r$ is $\operatorname{gcd}(n, r)$.

In the circulant graph $C_{10}(1,4,5),\left(v_{1}, v_{2}, \ldots, v_{10}\right)$ is a cycle of period 1 and length 10; cycles $\left(v_{1}, v_{5}, v_{9}, v_{3}, v_{7}\right)$ and $\left(v_{2}, v_{6}, v_{10}, v_{4}, v_{8}\right)$ are of period 4 and length $5=10 / \operatorname{gcd}(10,4)$ each; by considering the edges $v_{1} v_{16}, v_{2} v_{7}, v_{3} v_{8}, v_{4} v_{9}$, and $v_{5} v_{10}$ as double edges, they are cycles of period 5 and of length $2=10 / \operatorname{gcd}(10,5)$, each. See Figure 2 .

Theorem 3 (see $[15,18])$. If $C_{n}(R) \cong C_{n}(S)$, then there is a bijection $f$ from $R$ to $S$ so that for all $r \in R, \operatorname{gcd}(n, r)=$ $\operatorname{gcd}(n, f(r))$.

Proof. The proof is by induction on the order of $R$.

Remark 4. Let $|R|=k$. Then, the circulant graph $C_{n}(R)$ for a set $R=\left\{r_{1}, r_{2}, \ldots, r_{k}\right\}$ is $(2 k-1)$-regular if $n / 2 \in R$ and $2 k$-regular otherwise.

Definition 5. The cross product or Cartesian product of two simple graphs $G(V, E)$ and $H(W, F)$ is the simple graph $G \square H$ with vertex set $V \times W$ in which two vertices $u=\left(u_{1}, u_{2}\right)$ and $v=\left(v_{1}, v_{2}\right)$ are adjacent if and only if either $u_{1}=v_{1}$ and $u_{2} v_{2} \in F$ or $u_{2}=v_{2}$ and $u_{1} v_{1} \in E$.

Definition 6 (see $[4,13-18]$ ). Circulant graphs $C_{n}(R)$ and $C_{n}(S)$ for $R=\left\{r_{1}, r_{2}, \ldots, r_{k}\right\}$ and $S=\left\{s_{1}, s_{2}, \ldots, s_{k}\right\}$ are said to be Adam's isomorphic if there exists a positive integer $x$ relatively prime to $n$ with $\left\{s_{1}, s_{2}, \ldots, s_{k}\right\}=\left\{x r_{1}, x r_{2}, \ldots, x r_{k}\right\}_{n}^{*}$ where $\left\langle r_{i}\right\rangle_{n}^{*}$, the reflexive modular reduction of a sequence $\left\langle r_{i}\right\rangle$, is the sequence obtained by reducing each $r_{i}$ modulo $n$ to yield $r_{i}^{\prime}$ and then replacing all resulting terms $r_{i}^{\prime}$ which are larger than $n / 2$ by $n-r_{i}^{\prime}$.

The following lemmas are useful to obtain one-to-one mappings.

Lemma 7. Let $A$ and $B$ be two nonempty sets. Let $f: A \rightarrow B$ be a mapping. Then, $f$ is one-to-one if and only if $f / A^{\prime}$ is oneto-one for every nonempty subset $A^{\prime}$ of $A$.

Lemma 8. Let $A$ and $B$ be nonempty sets and let $A_{1}, A_{2}, \ldots, A_{k}$ be a partition of $A$ (each $A_{i}$ being nonempty), $i=1,2, \ldots, k$. Let $f: A \rightarrow B$ be a mapping. Then $f$ is one-to-one if and only if $f / A_{i}$ is one-to-one for every $i, i=1$, $2, \ldots, k$.

The motivation to do this research work is to develop a theory among circulant graphs similar to the product and factorization of natural numbers.

\section{Main Results}

Throughout this section, the following notation is used. Let $c \in \mathbb{N}$ and $A \subseteq \mathbb{N}$. Then $c A=\{c a: a \in A\}$. Let $[r, s]$ denote $\{r, r+1, \ldots, s\}, r \in \mathbb{Z}$, and let $n G$ denote disjoint union of $n$ copies of the graph G. $G \square H$ denotes the Cartesian product of graphs $G$ and $H$.

In this paper, Cartesian product and factorization of connected circulant graphs is studied. Moreover $C_{4}=P_{2} \square P_{2}$ and for $n \neq 4$ and nontrivial graphs $G$ and $H, C_{n} \neq G \square H$ and in particular, $C_{2 n+1}(R) \neq P_{2} \square G$ for any $R \subseteq[1, n]$.

In any circulant graph $C_{n}(R)$ the length of a periodic cycle of period $r$ is $n / \operatorname{gcd}(n, r)$. And 2, 3, 4, and 6 are the only numbers such that in any circulant graph $C_{n}(R)$ periodic 
cycle of length 2, 3, 4, or 6, if it exists, occurs without rotation always. This follows from the fact that for any natural number $m$ greater than 6 , there exists at least one natural number $s$ such that $1<s<[m / 2]$ and $\operatorname{gcd}(s, m)=1$ so that $C_{m} \cong C_{m}(s)$ and rotation of the periodic cycle of period $s$ is $s / \operatorname{gcd}(m, s)$. When $m=5, s=2$ is the required value. Thus, we call the periodic cycles of length 2, 3, 4, and 6 as the irrotational periodic cycles.

It is easy to verify the following:

(i) $G_{1} \square\left(G_{2} \cup G_{3}\right) \cong\left(G_{1} \square G_{2}\right) \cup\left(G_{1} \square G_{3}\right)$ for graphs $G_{1}$, $G_{2}$ and $G_{3}$. Here union is meant to be disjoint union only;

(ii) $G_{1} \square\left(C_{p m}(p R)\right) \cong G_{1} \square\left(p \cdot C_{m}(R)\right) \cong p\left(G_{1} \square C_{m}(R)\right)$;

(iii) $C_{p m}(p R) \square C_{q n}(q S) \cong p q\left(C_{m}(R) \square C_{n}(S)\right)$ for sets $R \subseteq$ $[1,[m / 2]], S \subseteq[1,[n / 2]], p, q \in \mathbb{N}$;

(iv) in any circulant graph $C_{n}(R)$ removal or addition of one or more jump sizes, if possible, will not change the property of being circulant;

(v) if $G$ and $H$ are nontrivial graphs of order $m$ and $n$, respectively, $m, n \geq 2$, then $G \square H$ contains $n$ number of disjoint copies of $G$ and $m$ number of disjoint copies of $H$.

Theorem 9. Let $n>2$. Then $P_{2} \square C_{n}$ is circulant if and only if $n$ is odd. Furthermore, in that case, $P_{2} \square C_{n} \cong C_{2 n}(2, n)$.

Proof. For $n>2, P_{2} \square C_{n}$ is a 3-regular connected graph of order $2 n$. When $n$ is even, $C_{2 n}(2, n) \cong 2 \cdot C_{n}(1, n / 2)$ which is a disconnected graph, whereas $P_{2} \square C_{n}$ is a connected one and hence $P_{2} \square C_{n} \neq C_{2 n}(2, n)$ when $n$ is even. For $n>2, P_{2} \square C_{n}$ contains two disjoint copies of $C_{n}$, say $C_{n}^{(1)}(1)$ and $C_{n}^{(2)}(1)$, so that $C_{n}^{(1)} \cup C_{n}^{(2)} \cong 2 \cdot C_{n}(1) \cong C_{2 n}(2)$ and $n$ disjoint copies of $P_{2}$. Let $C_{n}^{(j)}=\left(u_{0, j} u_{1, j} u_{2, j}, \ldots, u_{n-1, j}\right)$ and $u_{i, 1} u_{i, 2} \in E\left(P_{2} \square C_{n}\right)$, $i=0,1,2, \ldots, n-1$, and $j=1,2$. Thus $P_{2} \square C_{n} \cong C_{n}^{(1)} \cup$ $C_{n}^{(2)} \cup\left\{u_{i, 1} u_{i, 2} / i=0,1,2, \ldots, n-1\right\} \cong C_{2 n}(2) \cup\left\{u_{i, 1} u_{i, 2} / i=\right.$ $0,1,2, \ldots, n-1\}$ and $V\left(P_{2} \square C_{n}\right)=\left\{u_{i, j} / i=0,1,2, \ldots, n-1\right.$ and $j=1,2\}$.

Let $C_{2 n}(R)$ be a circulant graph with $n \in R$. In $C_{2 n}(R)$, cycle of period $n$ is of length 2 and irrotational. Using Remark 4, for $n>2$, 3-regular connected graph $P_{2} \square C_{n}$ is a circulant of the form $C_{2 n}(S)$ for some $S \subseteq[1, n]$ if and only if each edge $u_{i, 1} u_{i, 2}$ acts as a cycle of period $n$ (double edge) and equal number of vertices of $C_{n}^{(1)}$ (and of $C_{n}^{(2)}$ ) are on each sides of $u_{i, 1} u_{i, 2}, i=0,1,2, \ldots, n-1$. This is possible only when $n$ is odd, in which case the circulant graph is nothing but $C_{2 n}(2, n)$. The following transformation gives the required circulant graph representation of $P_{2} \square C_{n}$ when $n$ is odd.

Let $n=2 m+1, m \in \mathbb{N}$. Let $V\left(K_{2(2 m+1)}\right)=\left\{v_{0}, v_{1}, v_{2}, \ldots\right.$, $\left.v_{2(2 m+1)-1}\right\}$ where the vertices $v_{0}, v_{1}, v_{2}, \ldots, v_{2(2 m+1)-1}$ are considered as the corners of a regular $2(2 m+1)$-gon, assumed to be located in the plane proceeding cyclically clockwise. Define the mapping $\phi_{2,2 m+1}: V\left(P_{2} \square C_{2 m+1}\right) \rightarrow$ $V\left(K_{2(2 m+1)}\right)$ such that $\Phi_{2,2 m+1}\left(u_{i, j}\right)=v_{2 i+(j-1)(2 m+1)}$ and $\Phi_{2,2 m+1}((u, v))=\left(\Phi_{2,2 m+1}(u), \Phi_{2,2 m+1}(v)\right)$ for every $(u, v) \in E\left(P_{2} \square C_{2 m+1}\right)$, using subscript arithmetic modulo $2(2 m+1), j=1,2$ and $i=0,1, \ldots, 2 m$. Under this mapping, $\Phi_{2,2 m+1}\left(C_{2 m+1}^{(j)}\right)=\Phi_{2,2 m+1}\left(\left(u_{0, j} u_{1, j} u_{2, j} \ldots u_{2 m, j}\right)\right)=$ $\left(\Phi_{2,2 m+1}\left(u_{0, j}\right) \Phi_{2,2 m+1}\left(u_{1, j}\right) \Phi_{2,2 m+1}\left(u_{2, j}\right) \ldots \Phi_{2,2 m+1}\left(u_{2 m, j}\right)\right)=$ $\left(v_{(j-1)(2 m+1)} v_{2+(j-1)(2 m+1)} v_{4+(j-1)(2 m+1)} \ldots v_{2(2 m)+(j-1)(2 m+1)}\right)$, which is a periodic cycle of period 2 and of length $2 m+1$ in $C_{2(2 m+1)}(1,2, \ldots, 2 m+1)=K_{2(2 m+1)}, \Phi_{2,2 m+1}\left(\left(u_{i, 1}, u_{i+1,1}\right)\right)=$ $\left(v_{2 i}, v_{2 i+2}\right), \quad \Phi_{2,2 m+1}\left(\left(u_{i, 2}, u_{i+1,2}\right)\right)=\left(v_{2 i+2 m+1}, v_{2 i+2 m+3}\right)$ and $\Phi_{2,2 m+1}\left(\left(u_{i, 1}, u_{i, 2}\right)\right)=\left(v_{2 i}, v_{2 i+2 m+1}\right)$, using subscript arithmetic modulo $2(2 m+1), i=0,1,2, \ldots, 2 m$ and $j=1,2$. This implies that the mapping is a one-to-one mapping and preserves adjacency and the transformed graph $\Phi_{2,2 m+1}\left(P_{2} \square C_{2 m+1}\right)$ is $C_{2(2 m+1)}(2,2 m+1)=C_{2 n}(2, n)$, $n=2 m+1$ and $m \in \mathbb{N}$. Hence the result.

Note 1. Consider $P_{2} \square P_{2}=C_{4}$ which is a connected circulant graph. When $n$ is even and greater than $2, C_{2 n}(2, n) \cong 2 \cdot C_{n}(1$, $[n / 2])$, whereas $P_{2} \square C_{n}$ is connected but not circulant and hence $C_{2 n}(2, n) \neq P_{2} \square C_{n}$ when $n$ is even and greater than 2 .

Note 2. For $k \geq 3$ and $n \geq 2, P_{k} \square C_{n}$ is not circulant since it is not a regular graph.

Note 3. For $m>2$ and connected circulant $C_{m}(R), P_{2} \square$ $C_{m n}(n R) \cong n\left(P_{2} \square C_{m}(R)\right)$ since $H \square(n G) \cong n(H \square G)$ for any connected graphs $H$ and $G, n \in \mathbb{N}$.

Theorem 10. For $n \in \mathbb{N}, P_{2} \square C_{2 n+1}(R) \cong C_{2(2 n+1)}(2 R \cup\{2 n+$ $1\}) \cong C_{2(2 n+1)}(2 d R \cup\{2 n+1\})$, where $\operatorname{gcd}(2(2 n+1), d)=1$.

Proof. $P_{2} \square C_{2 n+1}(R)$ contains two disjoint copies of $C_{2 n+1}(R)$, say $G_{1}$ and $G_{2}$, and $n+1$ disjoint copies of $P_{2}$ so that $G_{1} \cup$ $G_{2} \cong 2 \cdot C_{2 n+1}(R) \cong C_{2(2 n+1)}(2 R)$ and $P_{2} \square C_{2 n+1}(R) \cong$ $C_{2(2 n+1)}(2 R) \cup n \cdot P_{2}$. Let $V\left(G_{j}\right)=\left(u_{0, j} u_{1, j} u_{2, j}, \ldots, u_{2 n, j}\right)$ and $u_{i, 1} u_{i, 2} \in E\left(P_{2} \square C_{2 n+1}(R)\right), i=0,1,2, \ldots, 2 n$ and $j=1,2$ so that $P_{2} \square C_{2 n+1}(R) \cong C_{2(2 n+1)}(2 R) \cup\left\{u_{i, 1} u_{i, 2} / i=0,1,2, \ldots, 2 n\right\}$ and $V\left(P_{2} \square C_{2 n+1}(R)\right)=\left\{u_{i, j} / i=0,1,2, \ldots, 2 n\right.$ and $\left.j=1,2\right\}$. At first let us assume that $C_{2 n+1}(R)$ is connected, $n \in \mathbb{N}$. Then, for every $r \in R$, the length of each periodic cycle of period $r$ in $C_{2 n+1}(R)$ is $(2 n+1) / \operatorname{gcd}(2 n+1, r)$, an odd number. Thus using the proof similar to that of Theorem 9 , equal number of vertices of each periodic cycle, starting from $u_{i, 1}$ or $u_{i, 2}$ of copies of $C_{2 n+1}(R)$, can be made on each sides of $u_{i, 1} u_{i, 2}$ in $P_{2} \square C_{2 n+1}(R)$, using the one-to-one mapping $\Phi_{2,2 n+1}: V\left(P_{2} \square C_{2 n+1}(R)\right) \rightarrow V\left(K_{2(2 n+1)}\right)$ defined by $\Phi_{2,2 n+1}\left(u_{i, j}\right)=v_{2 i+(j-1)(2 n+1)}$ and $\Phi_{2,2 n+1}((u, v))=\left(\Phi_{2,2 n+1}(u)\right.$, $\left.\Phi_{2,2 n+1}(v)\right)$ for every $(u, v) \in E\left(P_{2} \square C_{2 n+1}(R)\right)$, using subscript arithmetic modulo $2(2 n+1), i=0,1, \ldots, 2 n$, and $j=$ 1,2 where $V\left(K_{2(2 n+1)}\right)=\left\{v_{0}, v_{1}, v_{2}, \ldots, v_{2(2 n+1)-1}\right\}$ and the vertices $v_{0}, v_{1}, v_{2}, \ldots, v_{2(2 n+1)-1}$ are considered as the corners of a regular $2(2 n+1)$-gon assumed to be located in the plane proceeding cyclically clockwise. Under this transformation $\Phi_{2,2 n+1}\left(\left(u_{i, 1}, u_{i+r, 1}\right)\right)=\left(v_{2 i}, v_{2 i+2 r}\right), \Phi_{2,2 n+1}\left(\left(u_{i, 2}, u_{i+r, 2}\right)\right)=$ $\left(v_{2 i+2 n+1}, v_{2(i+r)+2 n+1}\right)$ and $\Phi_{2,2 n+1}\left(\left(u_{i, 1}, u_{i, 2}\right)\right)=\left(v_{2 i}, v_{2 i+2 n+1}\right)$, using subscript arithmetic modulo $2(2 n+1), r \in R$ and $i=0,1,2, \ldots, 2 n$. And the transformed graph is $C_{2(2 n+1)}(2 R \cup$ $\{2 n+1\}), n \in \mathbb{N}$. The above result is true also when $C_{2 n+1}(R)$ is disconnected, $n \in \mathbb{N}$. Hence $P_{2} \square C_{2 n+1}(R) \cong C_{2(2 n+1)}(2 R \cup$ $\{2 n+1\})$. When $\operatorname{gcd}(2(2 n+1), d)=1, d$ is an odd number, hence $d(2 n+1)=2 n+1$ under arithmetic modulo $2(2 n+1)$. 
Also $C_{2(2 n+1)}(2 R \cup\{2 n+1\})$ and $C_{2(2 n+1)}(2 d R \cup\{d(2 n+1)\})=$ $C_{2(2 n+1)}(2 d R \cup\{2 n+1\})$ are Adam's isomorphic graphs. Hence the result.

Corollary 11. For nonempty subset $R$ of $[1, n]$, if either $S \neq 2 d R \cup\{2 n+1\}$ for any d relatively prime to $2(2 n+1)$ under arithmetic modulo $2(2 n+1)$ or $C_{2 n+1}(R)$ is disconnected, then connected circulant $C_{2(2 n+1)}(S) \neq P_{2} \square C_{2 n+1}(R)$.

Proof. When $\Phi \neq R \subseteq[1, n]$ and $S=2 d R \cup\{2 n+1\}$ for any $d$ relatively prime to $2(2 n+1)$, then $C_{2(2 n+1)}(S)=P_{2} \square C_{2 n+1}(R)$, using Theorem 10. When $C_{2 n+1}(R)$ is disconnected, then the product graph $P_{2} \square C_{2 n+1}(R)$ is also disconnected whereas it is given that $C_{2(2 n+1)}(S)$ is connected. Hence the result.

Theorem 12. For $d, n \in \mathbb{N}$ and $\operatorname{gcd}(4(2 n+1), d)=1, C_{4} \square$ $C_{2 n+1}(S) \cong C_{4(2 n+1)}(4 S \cup\{2 n+1\}) \cong C_{4(2 n+1)}(4 d S \cup\{2 n+1\})$.

Proof. At first let us assume that $C_{2 n+1}(S)$ is connected. Let $G_{1}$, $G_{2}, G_{3}$, and $G_{4}$ be the four copies of $C_{2 n+1}(S)$ in $C_{4} \square C_{2 n+1}(S)$ so that $G_{1} \cup G_{2} \cup G_{3} \cup G_{4} \cong 4 \cdot C_{2 n+1}(S) \cong C_{4(2 n+1)}(4 S)$. Let $V\left(G_{i}\right)=\left\{u_{i, 1}, u_{i, 2}, \ldots, u_{i, 2 n+1}\right\},\left(u_{1, j} u_{2, j} u_{3, j} u_{4, j}\right)$ be the $j$ th copy of $C_{4}$ in $C_{4} \square C_{2 n+1}(S)$ and $V\left(C_{4} \square C_{2 n+1}(S)\right)=\left\{u_{i, j}:\right.$ $i=1,2,3,4$ and $j=1,2, \ldots, 2 n+1\}, i=1,2,3,4$ and $j=1,2, \ldots, 2 n+1$.

Let $V\left(K_{4(2 n+1)}\right)=\left\{v_{1}, v_{2}, \ldots, v_{4(2 n+1)}=v_{0}\right\}$ and the vertices $v_{0}, v_{1}, v_{2}, \ldots, v_{4(2 n+1)-1}$ are considered as the corners of a regular $4(2 n+1)$-gon assumed to be located in the plane proceeding cyclically clockwise. Define oneto-one mapping $\Phi_{4,2 n+1}: V\left(C_{4} \square C_{2 n+1}(S)\right) \rightarrow V\left(K_{4(2 n+1)}\right)$ such that $\Phi_{4,2 n+1}\left(u_{i, j}\right)=v_{(i-1)(2 n+1)+4(j-1)}$ using subscript arithmetic modulo $4(2 n+1)$ and $\Phi_{4,2 n+1}((u, v))=$ $\left(\Phi_{4,2 n+1}(u), \Phi_{4,2 n+1}(v)\right)$ for every $(u, v) \in E\left(C_{4} \square C_{2 n+1}(S)\right)$, $i=1,2,3,4$ and $j=1,2, \ldots, 2 n+1$. Then $\Phi_{4,2 n+1}\left(\left(u_{i, 1} u_{i, 2} u_{i, 3}\right.\right.$ $\left.\left.\ldots u_{i, 2 n+1}\right)\right)=\left(\Phi_{4,2 n+1}\left(u_{i, 1}\right) \Phi_{4,2 n+1}\left(u_{i, 2}\right) \Phi_{4,2 n+1}\left(u_{i, 3}\right) \ldots\right.$ $\left.\Phi_{4,2 n+1}\left(u_{i, 2 n+1}\right)\right)=\left(v_{0+(i-1)(2 n+1)} v_{4+(i-1)(2 n+1)} v_{8+(i-1)(2 n+1)} \cdots\right.$ $\left.v_{4(2 n)+(i-1)(2 n+1)}\right)=C_{2 n+1}^{(i)}$, say, and $\Phi_{4,2 n+1}\left(\left(u_{1, j} u_{2, j}\right.\right.$ $\left.\left.u_{3, j} \quad u_{4, j}\right)\right)=\left(v_{4(j-1)} v_{(2 n+1)+4(j-1)} v_{2(2 n+1)+4(j-1)} v_{3(2 n+1)+4(j-1)}\right)$ which is a periodic cycle of period $2 n+1$ and of length 4 in $C_{4(2 n+1)}(1,2, \ldots, 2(2 n+1))=K_{4(2 n+1)}$, using subscript arithmetic modulo $4(2 n+1), i=1,2,3,4$ and $j=1$, $2, \ldots, 2 n+1$. Also, $C_{2 n+1}^{(i)}$ is a periodic cycle of period 4 and of length $2 n+1$ in $C_{4(2 n+1)}(1,2, \ldots, 2(2 n+1))=K_{4(2 n+1)}$ since $v_{(i-1)(2 n+1)+4(j-1)}=v_{(i-1)(2 n+1)+4(k-1)}$ implies that $k=j, 1 \leq k, j \leq 2 n+1$, and $i=1,2,3,4$. Moreover, each cycle of period $s$ of $C_{2 n+1}(S)$ becomes periodic cycle of period $4 s$ in the transformed graph. Thus the transformed graph $\Phi_{4,2 n+1}\left(C_{4} \square C_{2 n+1}(S)\right)$ is nothing but $C_{4(2 n+1)}(R)$ where $R=4 S \cup\{2 n+1\}$. Also when $\operatorname{gcd}(4(2 n+1), d)=1, C_{4(2 n+1)}(4 S \cup\{2 n+1\})$ and $C_{4(2 n+1)}(4 d S \cup\{d(2 n+1)\})=C_{4(2 n+1)}(4 d S \cup\{2 n+1\})$ are Adam's isomorphic. Hence the result.

Theorem 13. Let $C_{2 n}(R)$ be connected, $n \in \mathbb{N}$. Then $C_{4} \square C_{2 n}(R) \neq C_{8 n}(S)$ for any $S \subseteq[1,4 n]$.

Proof. Using Theorem 9, $C_{4} \square C_{2}=C_{4} \square P_{2}$ is not circulant. For $n \geq 2$, if $C_{2 n}(R)$ is connected, then $C_{4} \square C_{2 n}(R)$ is also connected and it contains 4 disjoint copies of $C_{2 n}(R)$ and
$2 n$ number of disjoint copies of $C_{4}$. And hence if $C_{2 n}(R)$ is connected and $C_{4} \square C_{2 n}(R) \cong C_{8 n}(S)$ for some $S \subseteq$ $[1,4 n]$, then $S=4 k R \cup\{2 n d\}$ for some $k$ and $d$ such that $C_{8 n}(4 k R)=4 \cdot C_{2 n}(k R) \cong 4 \cdot C_{2 n}(R), C_{8 n}(2 n d)=$ $2 n \cdot C_{4}(d)$ and $\operatorname{gcd}(2 n, k)=1=\operatorname{gcd}(4, d)$. This implies $C_{4} \square C_{2 n}(R) \cong C_{8 n}(4 k R \cup\{2 d n\}) \cong 2 \cdot C_{4 n}(2 k R \cup\{d n\})$ which is disconnected, a contradiction when $C_{2 n}(R)$ is connected. Hence the result.

Lemma 14. Let $m, n \geq 2, r \in R$, and $s \in S$. If $C_{m}(r) \square C_{n}(s)$ is not circulant, then $C_{m}(R) \square C_{n}(S)$ is not circulant.

Proof. Let $r \in R$ and $s \in S$. Now, $C_{m}(r) \square C_{n}(s) \neq C_{m n}(T)$ for any $T \subseteq[1, m n / 2]$ implies that $C_{m}(R) \square C_{n}(s) \neq C_{m n}(U)$ for any $U \subseteq[1, m n / 2]$ (if not, let $r \in R$ and $C_{m}(R) \square C_{n}(s)=$ $C_{m n}(U)$ for at least one $U \subseteq[1, m n / 2]$. Then by removing all other jump sizes other than $r$ from $C_{m}(R)$, the resultant graph, $C_{m}(r) \square C_{n}(s)$, remains circulant, a contradiction to the given condition). Similarly we can prove that for $s \in$ $S$ if $C_{m}(R) \square C_{n}(s)$ is not circulant, then $C_{m}(R) \square C_{n}(S)$ is not circulant. Combining the above arguments, we get the result.

Theorem 15. Let $C_{2(2 n+1)}(R)$ be connected, $n \in \mathbb{N}$. Then $P_{2} \square C_{2(2 n+1)}(R)$ is circulant if and only if $C_{2(2 n+1)}(R) \cong P_{2} \square$ $C_{2 n+1}(S)$ for some $S \subseteq[1, n]$. Furthermore, in that case, $P_{2} \square C_{2(2 n+1)}(R) \cong C_{4} \square C_{2 n+1}(S) \cong C_{4(2 n+1)}(4 S \cup\{2 n+1\}) \cong$ $C_{4(2 n+1)}(4 d S \cup\{2 n+1\})$, where $\operatorname{gcd}(d, 4(2 n+1))=1$.

Proof. Let $C_{2(2 n+1)}(R) \cong P_{2} \square C_{2 n+1}(S)$. Then $C_{2(2 n+1)}(R) \cong$ $C_{2(2 n+1)}(2 S \cup\{2 n+1\})$, using Theorem 10. This implies that $P_{2} \square C_{2(2 n+1)}(R) \cong C_{4} \square C_{2 n+1}(S) \cong C_{4(2 n+1)}(4 S \cup\{2 n+1\}) \cong$ $C_{4(2 n+1)}(4 d S \cup\{2 n+1\})$, where $\operatorname{gcd}(d, 4(2 n+1))=1$, using Theorem 12 .

Conversely, let $P_{2} \square C_{2(2 n+1)}(R)$ be circulant. If $C_{2(2 n+1)}(R) \neq P_{2} \square C_{2 n+1}(S)$ for any connected $C_{2 n+1}(S)$, then $P_{2} \square C_{2(2 n+1)}(R)$ has $P_{2}$ as a factor and not $C_{4}=P_{2} \square P_{2}$. Let $R=2 S \cup T \subseteq[1,2 n+1]$ where $T \subseteq[1,2 n+1] \subset 2 \mathbb{N}+1$ and $T \neq \Phi$ since $C_{2(2 n+1)}(R)$ is connected. Let $2 r+1 \in T, r \in$ $\mathbb{N} \cup\{0\}$. Then using Theorem 2 the length of the periodic cycle of period $2 r+1$ in $C_{2(2 n+1)}(R)$ is $2(2 n+1) / \operatorname{gcd}(2(2 n+1), 2 r+1)$, an even number. Therefore $P_{2} \square C_{2(2 n+1)}(2 r+1)$ cannot be a circulant graph (see the proof of Theorem 9) and using Lemma 14, $P_{2} \square C_{2(2 n+1)}(R)$ cannot be a circulant graph, a contradiction to $P_{2} \square C_{2(2 n+1)}(R)$ is circulant. This implies that there exists a connected circulant $C_{2 n+1}(S)$ such that $C_{2(2 n+1)}(R) \cong P_{2} \square C_{2 n+1}(S)$. And thereby $P_{2} \square C_{2(2 n+1)}(R) \cong$ $C_{4} \square C_{2 n+1}(S) \cong C_{4(2 n+1)}(4 d S \cup\{2 n+1\})$ using Theorem 12 where $\operatorname{gcd}(d, 4(2 n+1))=1$. Hence the result.

Corollary 16. Let $n \geq 2$ and $C_{n}(R)$ be connected. Then $C_{4} \square C_{n}(R)$ is circulant if and only if $n$ is odd. Furthermore, in that case, $C_{4} \square C_{n}(R) \cong C_{4 n}(4 R \cup\{n\}) \cong C_{4 n}(4 d R \cup\{n\})$ where $\operatorname{gcd}(d, 4 n)=1$.

Proof. When $n$ is odd $C_{4} \square C_{n}(R) \cong C_{4 n}(4 d R \cup\{n\})$, using Theorem 12 where $\operatorname{gcd}(d, 4 n)=1$. Conversely, let $C_{4} \square C_{n}(R)$ be circulant, say $C_{4 n}(S)$ for some $S \subseteq[1,2 n]$. When $n$ is odd, the result is true by Theorem 12 . When $n$ is even, using Theorem 13, $C_{4} \square C_{n}(R)$ is not circulant. Hence the result. 
Corollary 17. Let $n \geq 2$ and $C_{n}(R)$ be connected. Then $C_{4} \square C_{n}(R)$ is circulant if and only if $n$ is odd if and only if $C_{4 n}(4 R \cup\{n\})$ is connected. Furthermore, in that case, $C_{4} \square$ $C_{2 n+1}(R) \cong C_{4(2 n+1)}(4 R \cup\{2 n+1\}) \cong C_{4(2 n+1)}(4 d R \cup\{2 n+1\})$ and $C_{4}(1,2) \square C_{2 n+1}(R) \cong C_{4(2 n+1)}(4 d R \cup\{2 n+1,2(2 n+1)\})$, where $\operatorname{gcd}(d, 4(2 n+1))=1$.

Theorem 18. Let $n>2$ and $C_{n}(R)$ be connected. Then $P_{2} \square C_{n}(R)$ is circulant if and only if $n$ is odd or $C_{n}(R) \cong$ $P_{2} \square C_{2 m+1}(S)$ for some connected $C_{2 m+1}(S), m \in \mathbb{N}$.

Proof. When $n$ is odd, $P_{2} \square C_{n}(R) \cong C_{2 n}(2 d R \cup\{n\})$, using Theorem 10 where $\operatorname{gcd}(d, 2 n)=1$. When $C_{n}(R) \cong$ $P_{2} \square C_{2 m+1}(S)$ for some connected $C_{2 m+1}(S), P_{2} \square C_{n}(R) \cong$ $C_{4} \square C_{2 m+1}(S) \cong C_{4(2 m+1)}(4 d S \cup\{2 m+1\})$, using Theorem 12 where $\operatorname{gcd}(d, 4(2 m+1))=1$.

Conversely, let $P_{2} \square C_{n}(R)$ be circulant. Consider the following two cases of $C_{n}(R)$.

Case i. $C_{n}(R) \cong P_{2} \square C_{k}(S)$ for some connected $C_{k}(S)$.

$C_{n}(R) \cong P_{2} \square C_{k}(S)$ is connected and $P_{2} \square C_{n}(R)$ is circulant implies that $P_{2} \square C_{n}(R) \cong P_{2} \square P_{2} \square C_{k}(S) \cong C_{4} \square C_{k}(S)$ is a connected circulant. This implies that $k$ is odd, using Corollary 16. Let $k=2 m+1$ so that $C_{n}(R) \cong P_{2} \square C_{k}(S)=$ $P_{2} \square C_{2 m+1}(S)$ for some connected $C_{2 m+1}(S), m \in \mathbb{N}$.

Case ii. $C_{n}(R) \neq P_{2} \square C_{2 m+1}(S)$ for any connected $C_{2 m+1}(S)$.

Our aim is to prove that $n$ is odd in this case. If not, let $n=$ $2 m, m \in \mathbb{N}$. Then $P_{2} \square C_{n}(R)=P_{2} \square C_{2 m}(R)$ is a connected circulant graph such that $C_{n}(R)=C_{2 m}(R) \neq P_{2} \square C_{2 k+1}(S)$ for any connected $C_{2 k+1}(S)$. This implies that $2 \mathrm{~m} / \operatorname{gcd}(2 \mathrm{~m}, r)$, the length of a periodic cycle of period $r$ in $C_{2 m}(R)$, is odd for all $r \in R$ since each copy of $P_{2}$ in the connected circulant graph $P_{2} \square C_{2 m}(R)$ acts as a periodic cycle of length 2 (see the proof of Theorem 9). This is possible only when $r$ is even for every $r \in R$. This implies that $R=2 R^{\prime} \subseteq[1, m]$ and thereby $C_{n}(R)=$ $C_{2 m}(R)=C_{2 m}\left(2 R^{\prime}\right)=2 \cdot C_{m}\left(R^{\prime}\right)$ which is not connected, a contradiction. This implies that $n$ must be odd. Hence the result.

Corollary 19. For $n>2$, the products $P_{2} \square C_{n}$ and $P_{2} \square K_{n}=$ $P_{2} \square C_{n}(1,2, \ldots,[n / 2])$ are circulant if and only if $n$ is odd. Furthermore, in that case, $P_{2} \square C_{2 n+1} \cong C_{2(2 n+1)}(2,2 n+1)$ and $P_{2} \square K_{2 n+1} \cong C_{2(2 n+1)}(2,4, \ldots, 2 n, 2 n+1) \subset K_{2(2 n+1)}, n \in \mathbb{N}$.

Proof. For $n>2, P_{2} \square C_{n}$ is circulant if and only if $n$ is odd, follows from Theorem 9. Using Theorem 10, $P_{2} \square K_{2 m+1}=$ $P_{2} \square C_{2 m+1}(1,2, \ldots, m) \cong C_{2(2 m+1)}(2\{1,2, \ldots, m\} \cup\{2 m+1\})=$ $C_{2(2 m+1)}(2,4, \ldots, 2 m, 2 m+1)$ which is a proper subgraph of $C_{2(2 m+1)}(1,2, \ldots, 2 m+1)=K_{2(2 m+1)}, m \in \mathbb{N}$. Now consider the case of $P_{2} \square K_{n}$ when $n$ is even. Let $n=2 m, m \in \mathbb{N}$. For $m \geq 2$, using Theorem 9, $P_{2} \square C_{2 m}=P_{2} \square C_{2 m}(1)$ is not a circulant graph and hence using Lemma 14, $P_{2} \square K_{2 m}$ is not a circulant graph. Hence the result.

Corollary 20. For $n>2, K_{n} \neq P_{2} \square C_{m}(S)$ and $K_{n} \neq C_{4} \square$ $C_{m}(S)$ for any connected circulant $C_{m}(S)$.

Proof. Clearly, for $n>2, K_{n}=C_{n}(1,2, \ldots,[n / 2])$, a connected circulant graph. If connected circulant graph
$K_{n}=C_{n}(1,2, \ldots,[n / 2]) \cong P_{2} \square C_{m}(S)$, then, using Theorem $18, m$ is odd or $C_{m}(S) \cong P_{2} \square C_{2 k+1}(R)$ for some connected circulant $C_{2 k+1}(R), k \in \mathbb{N}$. And in both cases, using Theorems 10 and 12, $P_{2} \square C_{m}(S)$ is a proper subgraph of $K_{2 m}=$ $K_{n}$ and $\neq K_{n}$, a contradiction for $m>1$. This implies that $K_{n} \neq P_{2} \square C_{m}(S)$ for any connected circulant $C_{m}(S), n>2$. Similarly if connected circulant graph $C_{n}(1,2, \ldots,[n / 2])=$ $K_{n} \cong C_{4} \square C_{m}(S)$, then, using Corollary $16, m$ is odd and $C_{4} \square C_{m}(S)=C_{4} \square C_{2 k+1}(S) \cong C_{4(2 k+1)}(4 d S \cup\{2 k+1\})=$ $C_{4 m}(4 d S \cup\{m\})$ which is a proper subgraph of $K_{4 m}=K_{n}$ and $\neq K_{n}$, a contradiction where $m=4 k+1$ and $\operatorname{gcd}(4 m, d)=1$, $k, d \in N$. Hence the result.

So far we could find out when the cross product of $P_{2}$ or $\mathrm{C}_{4}$ with another circulant is also circulant. It is interesting to know, in general, whether the cross product of any two connected circulant graphs is circulant or not. If so, when is it circulant? The following give some positive results in this direction.

Theorem 21. For $m, n>2, C_{m} \square C_{n} \cong C_{m n}(m, n)$ if and only if $\operatorname{gcd}(m, n)=1$.

Proof. Let $m, n>$ 2. At first, assume that $C_{m} \square C_{n} \cong$ $C_{m n}(m, n) . C_{m} \square C_{n}$ is a connected graph which implies that $C_{m n}(m, n)$ is also connected. This implies, using Theorem 1 , $\operatorname{gcd}(m n, m, n)=1$ which implies $\operatorname{gcd}(m, n)=1$.

Conversely, let $\operatorname{gcd}(m, n)=1$. Without loss of generality, let us assume that $2<m<n$. Now $C_{m} \square C_{n}$ is a connected 4regular graph containing $m$ disjoint copies of $C_{n}$, $(n$ disjoint copies of $C_{m}$ ) and through all the $m$ isomorphic images of each vertex of $C_{n}$, there exists a cycle of length $m$. And for all possible values of $m$ and $n, n \cdot C_{m} \cong C_{m n}(n)$ and $m$. $C_{n} \cong C_{m n}(m)$ are the two edge disjoint spanning circulant subgraphs of $C_{m} \square C_{n}$.

Claim. $C_{m} \square C_{n} \cong C_{m n}(m, n)$.

$C_{m} \square C_{n}$ contains $m$ copies of $C_{n}$ and $C_{m n}(m) \cong m$. $C_{n}(1)$ is a spanning subgraph of $C_{m} \square C_{n}$. Let $C_{n}^{(i)}=$ $\left(\begin{array}{llll}u_{i, 1} & u_{i, 2} \ldots & u_{i, n}\end{array}\right)$ be the $i$ th copy of $C_{n}$ in $C_{m} \square C_{n}$ where $u_{i, j}$ is the isomorphic image of $u_{1, j}$ of $C_{n}^{(1)}$ in $C_{n}^{(i)}$ so that $\left(u_{1, j} u_{2, j} \ldots u_{m, j}\right)$ is a cycle of length $m$ in $C_{m} \square C_{n}$ and $V$ $\left(C_{m} \square C_{n}\right)=\left\{u_{i, j}: i=1,2, \ldots, m\right.$, and $\left.j=1,2, \ldots, n\right\}$, $j=1,2, \ldots, n$ and $i=1,2, \ldots, m$. Let $V\left(K_{m n}\right)=\left\{v_{1}, v_{2}, \ldots\right.$, $\left.v_{m n}=v_{0}\right\}$. Vertices $v_{0}, v_{1}, v_{2}, \ldots, v_{m n-1}$ are considered as the ordered vertices of a regular $m n$-gon, assumed to be located in the plane, proceeding cyclically clockwise. Define a mapping $\Phi_{m, n}: V\left(C_{m} \square C_{n}\right) \rightarrow V\left(C_{m n}(1,2, \ldots, m n / 2)\right)=V\left(K_{m n}\right)$ such that $\Phi_{m, n}\left(u_{i, j}\right)=v_{(j-1) m+(i-1) n}$ and $\Phi_{m, n}((u, v))=$ $\left(\Phi_{m, n}(u), \Phi_{m, n}(v)\right)$ for every $(u, v) \in E\left(C_{m} \square C_{n}\right)$, using subscript arithmetic modulo $m n, 1 \leq i \leq m$ and $1 \leq j \leq n$. Under this transformation $\Phi_{m, n}\left(C_{n}^{(i)}\right)=\Phi_{m, n}\left(\left(u_{i, 1} u_{i, 2} u_{i, 3} \ldots u_{i, n}\right)\right)=$ $\left(\Phi_{m, n}\left(u_{i, 1}\right) \Phi_{m, n}\left(u_{i, 2}\right) \Phi_{m, n}\left(u_{i, 3}\right) \ldots \Phi_{m, n}\left(u_{i, n}\right)\right) \stackrel{1}{=}\left(v_{(i-1) n}\right.$ $\left.v_{(i-1) n+m} v_{(i-1) n+2 m} \ldots v_{(i-1) n+(n-1) m}\right)$ which is a periodic cycle of $\left(\right.$ period $m$ ) length $m n / \operatorname{gcd}(m n, m)=n$ in $C_{m n}$ $(1,2, \ldots, m n / 2), i=1,2, \ldots, m$ and these $m$ cycles are vertex disjoint periodic cycles in $C_{m n}(1,2, \ldots, m n / 2)$. Similarly, $\phi_{m, n}\left(\left(u_{1, j} u_{2, j} u_{3, j} \cdots u_{i, j} \cdots u_{m, j}\right)\right)=\left(v_{(j-1) m}\right.$ $\left.v_{(j-1) m+n} v_{(j-1) m+2 n} \ldots v_{(j-1) m+(m-1) n}\right)$ which is a periodic cycle 


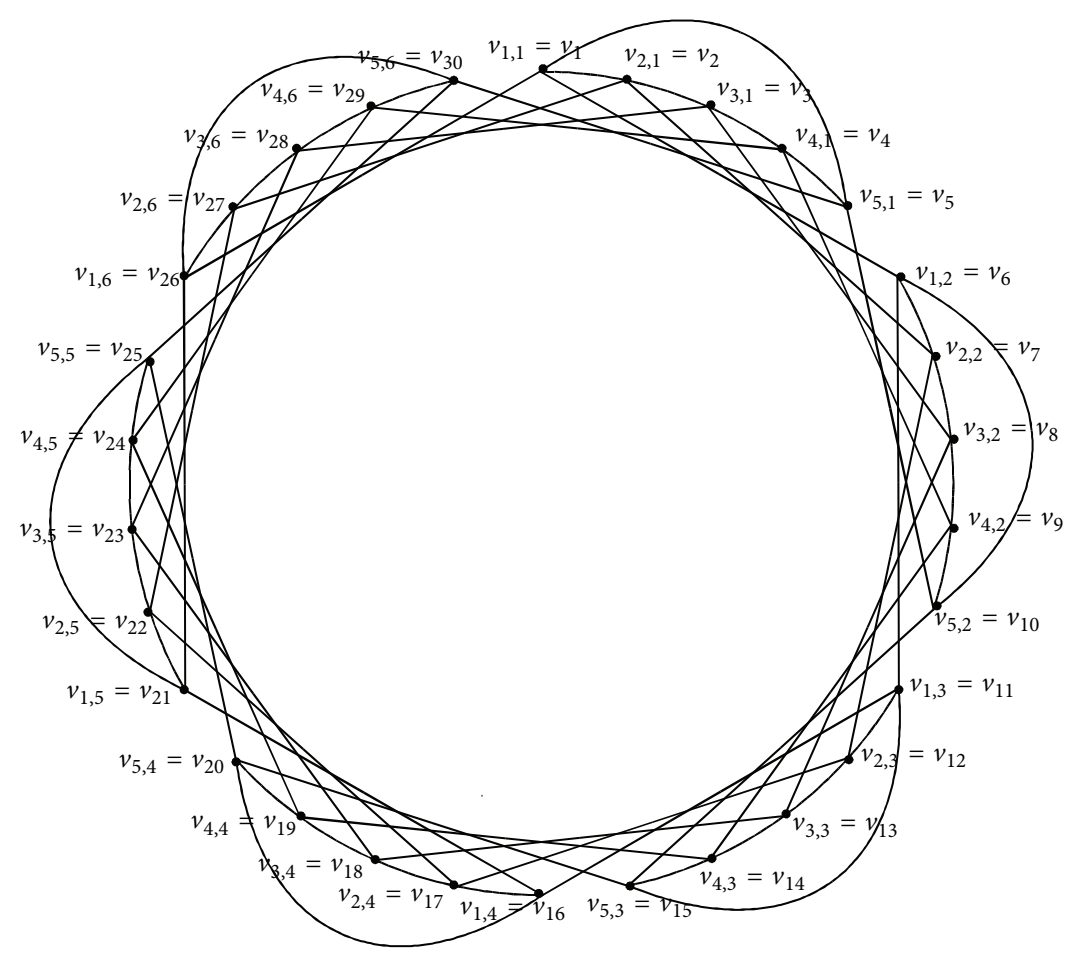

Figure 3: $C_{5} \times C_{6}$.

of period $n$ and length $m$ in $C_{m n}(1,2, \ldots, m n / 2), j=$ $1,2, \ldots, n$, and these $n$ cycles are vertex disjoint periodic cycles in $C_{m n}(1,2, \ldots, m n / 2)$ (transformation $\Phi_{m, n}$ is similar to $\theta_{n, r, t}$ defined in [13-18] to define type-2 isomorphism of circulant graphs). And the edges of $C_{m} \square C_{n}$ are the edges of $n$ disjoint copies of $C_{m}$ and $m$ disjoint copies of $C_{n}$ only. Clearly, $\Phi_{m, n}$ is a one-to-one mapping and preserves adjacency and $\Phi_{m, n}\left(C_{m} \square C_{n}\right)=C_{m n}(m, n)$. Here the mapping $\Phi_{m, n}$ is oneto-one that follows from Lemma 8 once by considering each $C_{n}^{(i)}=A_{i}$ and $A=V\left(C_{m} \square C_{n}\right)$ and in another by considering each $C_{m}^{(j)}=A_{j}$ and $A=V\left(C_{m} \square C_{n}\right), i=1,2, \ldots, m$ and $j=1,2, \ldots, n$. The transformation $\Phi_{m, n}$ defined on $C_{5} \square C_{6}$ is illustrated in Figures 3 and 4. $C_{5} \square C_{6}$ is given in Figure 3 and $\Phi_{5,6}\left(C_{5} \square C_{6}\right)=C_{30}(5,6)$ is given in Figure 4.

Corollary 22. Let $m, n>2$. Then $C_{m} \square C_{n}$ is circulant if and only if $\operatorname{gcd}(m, n)=1$ if and only if $C_{m} \square C_{n} \cong C_{m n}(d m, d n)$, where $\operatorname{gcd}(m n, d)=1$.

Proof. Using Theorem 21, for $m, n>2, C_{m} \square C_{n} \cong C_{m n}(m, n)$ if and only if $\operatorname{gcd}(m, n)=1$. Moreover, if $C_{n}(p, q)$ and $C_{n}(r, s)$ are isomorphic, then they are Adam's isomorphic only $[18,19]$ and, when $\operatorname{gcd}(n, d)=1, C_{n}(p, q)$ and $C_{n}(d p, d q)$ are Adam's isomorphic circulant graphs. Hence $C_{m} \square C_{n} \cong C_{m n}(m, n) \cong$ $C_{m n}(d m, d n)$, where $\operatorname{gcd}(m, n)=1=\operatorname{gcd}(m n, d)$.

Note 4. For any integer $d$ relatively prime to $m n$ and for a set $R \subseteq[1, m n / 2], C_{m n}(R)$ and $C_{m n}(d R)$ are Adam's isomorphic. Thus when $\operatorname{gcd}(m, n)=1=\operatorname{gcd}(m n, d), C_{m} \square C_{n} \cong$ $C_{m n}(m, n) \cong C_{m n}(d m, d n), d \in \mathbb{N}$. Thus $P_{2} \square C_{2 n+1} \cong$ $C_{2(2 n+1)}(2,2 n+1), C_{5} \square C_{6} \cong C_{30}(5,6)$, and $C_{7} \square C_{10} \cong$

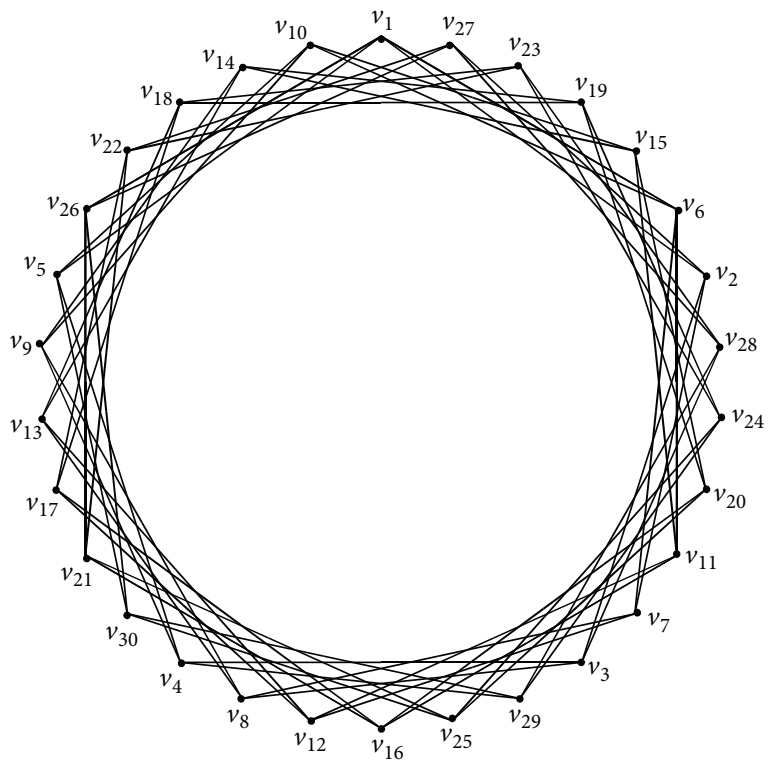

FIGURE 4: Transformed graph of $C_{5} \times C_{6} \cong C_{30}(5,6)$.

$C_{70}(7,10)$. Also $C_{5} \square C_{6} \cong C_{30}(5,6) \cong C_{30}(7 \times 5,7 \times 6) \cong$ $C_{30}(5,12) \cong C_{30}(5,7 \times 6)$ and $C_{7} \square C_{10} \cong C_{70}(7,10) \cong$ $C_{70}(9 \times 7,9 \times 10) \cong C_{70}(7,20) \cong C_{70}(7,9 \times 10)$.

Corollary 23. Let $a, b, m, n \in \mathbb{N}, 1 \leq 2 r+1 \leq 2 m+1$ and $1 \leq 2 s+1 \leq 2 n+1$. Then $C_{2^{a}(2 m+1)}(2 r+1) \square C_{2^{b}(2 n+1)}(2 s+1)$ is not circulant. 
Proof. Let $\operatorname{gcd}(2 m+1,2 r+1)=2 x+1$ and $\operatorname{gcd}(2 n+1,2 s+$ $1)=2 y+1, x, y \in \mathbb{N} \cup\{0\}$. This implies $\operatorname{gcd}((2 m+1) /(2 x+$ $1),(2 r+1) /(2 x+1))=1=\operatorname{gcd}\left(2^{a}(2 m+1) /(2 x+1),(2 r+\right.$ $1) /(2 x+1))$, and $\operatorname{gcd}((2 n+1) /(2 y+1),(2 s+1) /(2 y+1))=$ $1=\operatorname{gcd}\left(2^{b}(2 n+1) / 2 y+1,2 s+1 / 2 y+1\right)$ which implies that $C_{2^{a}(2 m+1)}(2 r+1) \cong(2 x+1) \cdot C_{2^{a}(2 m+1) /(2 x+1)}((2 r+1) /(2 x+1)) \cong$ $(2 x+1) \cdot C_{2^{a}(2 m+1) / 2 x+1} \cong(2 x+1) \cdot C_{2^{a}\left(2 m^{\prime}+1\right)}$ and similarly $C_{2^{b}(2 n+1)}(2 s+1) \cong(2 y+1) \cdot C_{2^{b}\left(2 n^{\prime}+1\right)}$ for some $m^{\prime}, n^{\prime} \in \mathbb{N}$ where $(2 m+1) /(2 x+1)=2 m^{\prime}+1$ and $(2 n+1) /(2 y+1)=2 n^{\prime}+1$. This implies that $C_{2^{a}(2 m+1)}(2 r+1) \square C_{2^{b}(2 n+1)}(2 s+1) \cong(2 x+$ 1) $(2 y+1) \cdot C_{2^{a}\left(2 m^{\prime}+1\right)} \square C_{2^{b}\left(2 n^{\prime}+1\right)}$, which is not circulant using Theorem 21 since $\operatorname{gcd}\left(2^{a}\left(2 m^{\prime}+1\right), 2^{b}\left(2 n^{\prime}+1\right)\right) \geq 2, m^{\prime}, n^{\prime} \in \mathbb{N}$. Hence the result.

Corollary 24. Let $C_{k^{a} m}(R)$ and $C_{k^{b} n}(S)$ be connected, $m, n>$ $2, k \geq 1, \operatorname{gcd}(m, n)=1=\operatorname{gcd}(m n, k)$ and $a, b \in \mathbb{N} \cup\{0\}$. Then $C_{m}(R) \square C_{k^{b} n}(S)$ and $C_{2^{a+1} m}(R) \square C_{2^{b+1} n}(S)$ are circulants when $C_{2^{a+1} m}(R)=C_{2 m}(R) \cong P_{2} \square C_{m}\left(T_{1}\right)$ and $C_{2^{b+1} m}(R)=C_{2 n}(S) \cong$ $P_{2} \square C_{n}\left(T_{2}\right)$ for some $T_{1}$ and $T_{2}$.

Corollary 25. For $a, b, k, m, n \in \mathbb{N}, m, n>2, k \geq 2$, $\operatorname{gcd}(m, n)=1, k^{a+b} \neq 4$ and connected circulants $C_{n}(R), C_{n}(S)$, $C_{k^{a} m}(R)$, and $C_{k^{b} n}(S), C_{n}(R) \square C_{n}(S)$ and $C_{k^{a} m}(R) \square C_{k^{b} n}(S)$ are not circulant.

In the proof of Theorem 21, we have, when $m, n>2$ and $\operatorname{gcd}(m, n)=1, C_{m} \square C_{n} \cong C_{m n}(m, n) \cong C_{m n}(d m, d n)$ where $\operatorname{gcd}(m n, d)=1$. In the transformation $\Phi_{m, n}$ defined on the graph $C_{m} \square C_{n}$ each copy of the cycle $C_{n}$ is rotated uniformly (and thereby the relative positions of the vertices of each copy of $C_{n}$ are not changed) with respect to the regular $m n$-gon so as to make uniform rotation of each copy of $C_{m}$ with respect to the regular $m n$-gon. Here any vertex $u_{i, j} \rightarrow v_{(j-1) m+(i-1) n}$ using subscript arithmetic modulo $m n, i=1,2, \ldots, m$ and $j=1,2, \ldots, n$. Thereby, for $m, n>2, \operatorname{gcd}(m, n)=1$ and connected circulant $C_{m}(R)$ and $C_{n}(S), \Phi_{m, n}\left(C_{m}(R) \square C_{n}\right) \cong$ $\Phi_{n, m}\left(C_{n} \square C_{m}(R)\right) \cong C_{m n}(n R \cup\{m\}) \cong C_{m n}(d n R \cup\{d m\})$ and $\Phi_{m, n}\left(C_{m} \square C_{n}(S)\right) \cong C_{m n}(m S \cup\{n\}) \cong C_{m n}(d m S \cup\{d n\})$ where $\operatorname{gcd}(m n, d)=1$. And if we introduce jump sizes $r_{1}, r_{2}, \ldots, r_{j}$ instead of jump size 1 in $C_{m}$ and $s_{1}, s_{2}, \ldots, s_{k}$ instead of 1 in $C_{n}$ and making the above transformation on $C_{m}(R) \square C_{n}(S)$, we get $\Phi_{m, n}\left(C_{m}(R) \square C_{n}(S)\right)=C_{m n}(n R \cup m S) \cong C_{m n}(d n R \cup$ $d m S)$, where $C_{m}(R)$ and $C_{n}(S)$ are connected, $m, n>2$, $\operatorname{gcd}(m, n)=1, \operatorname{gcd}(m n, d)=1, R=\left\{r_{1}, r_{2}, \ldots, r_{j}\right\}$, and $S=\left\{s_{1}, s_{2}, \ldots, s_{k}\right\}$. Thus, we obtain the following result.

Theorem 26. Let $C_{m}(R)$ and $C_{n}(S)$ be connected, $m, n>2$ and $\operatorname{gcd}(m, n)=1=\operatorname{gcd}(m n, d)$. Then $C_{m}(R) \square C_{n}(S) \cong$ $C_{m n}(m S \cup n R) \cong C_{m n}(d m S \cup d n R)$.

Theorem 27. Let $m, n \in \mathbb{N}$ and $C_{2 m+1}(R)$ and $C_{2 n+1}(S)$ be connected circulant graphs. Then $C_{2 m+1} \square C_{2 n+1}$ is circulant if and only if $K_{2 m+1} \square K_{2 n+1}$ is circulant if and only if $C_{2 m+1}(R) \square C_{2 n+1}(S)$ is circulant if and only $\operatorname{gcd}(2 m+1,2 n+$ $1)=1$. Furthermore, in that case, there exists natural number d relatively prime to $(2 m+1)(2 n+1)$ such that $C_{2 m+1}(R) \square C_{2 n+1}(S) \cong C_{(2 m+1)(2 n+1)}((2 n+1) R \cup(2 m+1) S) \cong$ $C_{(2 m+1)(2 n+1)}(d(2 n+1) R \cup d(2 m+1) S)$.
Theorem 28. Let $G$ be a connected graph of order $n, n>2$. Then $P_{2} \square G$ is circulant if and only if $G \cong H$ or $P_{2} \square H$ where $H$ is a connected circulant graph of odd order.

Proof. Let $G \cong H$ or $P_{2} \square H$ for some connected circulant graph $H$ of odd order. Then, by Theorem 18, $P_{2} \square G$ is circulant.

Conversely, let $P_{2} \square G \cong C_{2 n}(S)$ be a connected circulant of order $2 n$ for some $S \subseteq[1, n], n>2$. Clearly, $P_{2} \square G \cong$ $C_{2 n}(S) \subseteq P_{2} \square K_{n}, n>2$. Now, consider different cases of $G$.

Case i. $O(G)=n=2 m+1, m \in N$.

Clearly, $G \subseteq K_{2 m+1}$ and, using Corollary 19, $P_{2} \square G \cong$ $C_{2 n}(S)=C_{2(2 m+1)}(S) \subseteq P_{2} \square K_{2 m+1} \cong C_{2(2 m+1)}(2,4, \ldots$, $2 m, 2 m+1)$. This implies that $S \subseteq 2 T \cup\{2 m+1\}$, where $T=[1, m]$ and $S \neq \phi . S=\{2 m+1\}$ and $S \subseteq 2 T$ are not possible since $C_{2(2 m+1)}(S)$ is connected. When $S=2 T^{\prime} \cup\{2 m+$ $1\}, P_{2} \square G \cong C_{2(2 m+1)}\left(2 T^{\prime} \cup\{2 m+1\}\right) \cong P_{2} \square C_{2 m+1}\left(T^{\prime}\right)$, using Theorem 10 where $\phi \neq T^{\prime} \subseteq T=[1, m]$. Thus in this case, we get $G \cong C_{2 m+1}\left(T^{\prime}\right)$ for some connected circulant $C_{2 m+1}\left(T^{\prime}\right)$.

Case ii. $G \cong P_{2} \square H$ where $H$ is a connected circulant graph of order $2 m+1, m \in \mathbb{N}$.

Then, $P_{2} \square G$ is circulant which implies that $C_{4} \square H$ is circulant. Clearly, using Corollary 17, $P_{2} \square G \cong C_{4} \square H \cong$ $C_{4(2 m+1)}(S) \subseteq C_{4} \square K_{2 m+1} \cong C_{4(2 m+1)}(4 d, 8 d, \ldots, 4 m d, 2 m+1)$ for some $S \subseteq[1,2(2 m+1)]$ and every $d$ relative prime to $4(2 m+1)$. This implies that $S \subseteq 4 d T \cup\{2 m+1\}, d \in \mathbb{N}$, $\operatorname{gcd}(4(2 m+1), d)=1, T=[1, m]$, and $S \neq \phi . S=\{2 m+1\}$ and $S \subseteq 4 d T$ are not possible since $C_{4(2 m+1)}(S)$ is connected. When $S=4 d T^{\prime} \cup\{2 m+1\}$ for some $T^{\prime}$ and $d \in \mathbb{N}$ such that $\operatorname{gcd}(4(2 m+1), d)=1, \Phi \neq T^{\prime} \subseteq T$, then $P_{2} \square G \cong$ $C_{4} \square H \cong C_{4(2 m+1)}(S) \cong C_{4(2 m+1)}\left(4 d T^{\prime} \cup\{2 m+1\}\right) \cong$ $C_{4} \square C_{2 m+1}\left(T^{\prime}\right)$ using Theorem 15. This implies $G \cong P_{2} \square$ $C_{2 m+1}\left(T^{\prime}\right)$ for some connected circulant $C_{2 m+1}\left(T^{\prime}\right)$.

Also, $P_{2} \square G \cong C_{2 n}(S)$, a circulant graph implies that $2 G$ and $n \cdot P_{2}$ are two edge-disjoint spanning subgraphs of $C_{2 n}(S)$. This implies if we remove all the $n$ copies of circulant subgraph $P_{2}=C_{2}$ from the circulant graph $C_{2 n}(S) \cong P_{2} \square G$, then the resultant graph $2 G$ must be a circulant graph (in any circulant graph removal or addition of one or more jump sizes, if possible, will not change the property of being circulant) which implies that $G$ is circulant. Now $G$ and $P_{2} \square G$ are circulants and using Theorem 18 the graph $G$ is either of odd order or product of $P_{2}$ and an odd order circulant graph. Hence the result.

Theorem 29. Let $G$ be a connected graph of order $n \geq 2$. Then $C_{4} \square G$ is circulant if and only if $G$ is circulant of odd order.

Proof. When $G$ is a connected circulant of odd order, then using Corollary 19, $C_{4} \square G$ is circulant. Conversely, let $C_{4} \square G$ be a connected circulant, say $C_{4 n}(R)$. In any circulant graph $C_{4 n}(R)$ periodic cycles, each of length 4 occur without rotation. The spanning subgraph $n \cdot C_{4}$ is also a circulant subgraph in $C_{4 n}(R) \cong C_{4} \square G$. And hence if we remove all the edges of the spanning circulant subgraph $n \cdot C_{4}$ from the circulant graph $C_{4 n}(S) \cong C_{4} \square G$, then the resultant graph $4 G$ must 
be a circulant graph which implies that $G$ is circulant. When $G$ and $C_{4} \square G$ are connected circulant graphs, then, using Corollary 19, the order of $G$ is odd. Hence the result.

Theorem 30. If $G$ and $H$ are connected graphs and $G \square H$ is circulant, then $G$ and $H$ are circulants.

Proof. Let $G$ and $H$ be connected graphs of order $m$ and $n$, respectively, $m, n \in \mathbb{N}$. Then the order of the graph $G \square H$ is $m n$. Let $G \square H$ be circulant. For $m$ or $n=1$ or $m=$ $n=2$, the result is true. Now let $2 \leq m \leq n$. When at least one of the two graphs, say $G$, is circulant, then $n G$ is a spanning subgraph which is also a circulant subgraph of the circulant graph $G \square H$. If we remove all the edges of spanning circulant subgraph $n G$ from the circulant graph $G \square H$ the resultant graph $m H$ is circulant which implies that $H$ is circulant. When both the graphs $G$ and $H$ are not circulant, then let $C_{m}\left(R_{1}\right)$ be a spanned subgraph of $G$ obtained from $G$ by removal of minimum number of edges and $C_{m}\left(R_{2}\right)$ be a circulant graph which is obtained from $G$ by adding minimum number of edges in $G$. Similarly let $C_{n}\left(S_{1}\right)$ and $C_{n}\left(S_{2}\right)$ be the corresponding circulant graphs obtained with respect to the graph $H$. This implies that $C_{m}(\Phi) \square C_{n}(\Phi) \subseteq$ $C_{m}\left(R_{1}\right) \square C_{n}\left(S_{1}\right) \subset G \square H \subset C_{m}\left(R_{2}\right) \square C_{n}\left(S_{2}\right) \subseteq K_{m} \square K_{n}$ which implies, from the construction, that $G \square H \neq$ product of a circulant graph of order $m$ and a circulant graph of order $n$ which implies $G \square H$ is not a circulant graph, a contradiction to the given condition that the graph $G \square H$ is circulant. This implies $G$ and $H$ are circulant graphs. Hence the result.

Theorem 31. Let $G$ and $H$ be connected graphs, each of order $>2$. Then $G \square H$ is circulant if and only if $G$ and $H$ are circulants and satisfy one of the following conditions:

(i) $G \cong C_{m}(R) ; H \cong C_{n}(S)$ and $\operatorname{gcd}(m, n)=1$;

(ii) $G \cong C_{2 m+1}(R) ; H \cong C_{2 n+1}(S), P_{2} \square C_{2 n+1}(S), C_{4} \square$ $C_{2 n+1}(S)$ or $C_{2^{k}(2 n+1)}(S)$ and $\operatorname{gcd}\left(2 m+1,2^{k}(2 n+1)\right)=$ $1, k \in \mathbb{N}$;

(iii) $G \cong P_{2} \square C_{2 m+1}(R) ; H \cong C_{2 n+1}(S)$ or $P_{2} \square C_{2 n+1}(S)$ and $\operatorname{gcd}(2 m+1,2 n+1)=1$;

(iv) $G \cong C_{2^{k}(2 m+1)}(R) \neq P_{2} \square C_{2^{k-1}(2 m+1)}(T)$ for any $C_{2^{k-1}(2 m+1)}(T) ; H \cong C_{2 n+1}(S)$ and $\operatorname{gcd}\left(2^{k}(2 m+1), 2 n+\right.$ $1)=1, k \in \mathbb{N}$;

(v) $G \cong C_{4} \square C_{2 m+1}(R) ; H \cong C_{2 n+1}(S)$ and $\operatorname{gcd}(2 m+$ $1,2 n+1)=1$;

(vi) $G \cong C_{2^{k}(2 m+1)}(R) \neq C_{4} \square C_{2^{k-2}(2 m+1)}(T), P_{2} \square C_{2^{k-1}(2 m+1)}$ $(U)$ for any $C_{2^{k-2}(2 m+1)}(T)$ and $C_{2^{k-1}(2 m+1)}(U) ; H \cong$ $C_{2 n+1}(S)$ and $\operatorname{gcd}\left(2^{k}(2 m+1), 2 n+1\right)=1, k \in \mathbb{N}, k \geq 2$.

Proof. Let $G$ and $H$ be of order $m$ and $n$, respectively, $m, n>2$. Then $G \square H$ is a connected graph of order $m n, m n>4$. Graph $G \square H$ is circulant if and only if $G$ and $H$ are circulants that follow from Theorem 30. Let $G \cong C_{m}(R)$ and $H \cong C_{n}(S)$ for some $R \subseteq[1, m / 2]$ and $S \subseteq[1, n / 2]$. Then the rest of the result follows from Theorems 10, 12, and 26 and Corollaries 17, 24, and 25 .
Corollary 32. $P_{2} \square C_{4} \square G$ and $C_{4} \square P_{2} \square H$ are not circulant for any graphs $G$ and $H$.

Proof. $P_{2} \square C_{4}$ is not circulant using Theorem 18. Then the result follows from Theorem 31 .

Definition 33 (see [21]). A nontrivial graph $G$ is said to be prime if $G=G_{1} \square G_{2}$ implies that $G_{1}$ or $G_{2}$ is trivial; $G$ is composite if it is not prime.

Sabidussi [20] proved that every nontrivial graph is the unique product of prime graphs. In view of this and of the result that $m \cdot C_{n}(R) \cong C_{m n}(m R)$, let us look into the definition of prime circulant graphs.

Definition 34. If $C_{m}(R), C_{n}(S)$ and $C_{m n}(T)$ are circulant graphs such that $C_{m}(R) \square C_{n}(S) \cong C_{m n}(T)$, then we say that $C_{m}(R)$ and $C_{n}(S)$ are divisors or factors of $C_{m n}(T)$.

Thus for any connected circulant graph, the graph and $C_{1}()=K_{1}$ are always divisors and so we call them as improper divisors of the circulant graph. Divisors which are integer multiple of improper divisors also are called as improper divisors of the circulant graph. This does not arise since we consider divisors of connected graphs only. Divisor(s) other than improper divisors is called proper divisor(s) of the circulant graph.

Definition 35. A circulant graph whose only divisors are improper is called a prime circulant graph. Other circulant graphs are called composite circulant graphs.

In view of Corollary 32, for any connected circulant, both $P_{2}$ and $C_{4 m}(R)$ cannot be proper divisors, $m \in N ; C_{4}=$ $P_{2} \square P_{2}=C_{2} \square C_{2}$ and we consider $C_{2}=P_{2}$ as a prime circulant.

It is easy to verify the following result using Theorems 9, 10, 15, 18, 27, 28, 29, and 31, and Corollaries 17, 24, and 25.

Theorem 36 (factorization theorem on circulant graphs). Let $m$ and $n$ be relatively prime integers. If $R \subseteq[1, m / 2], S \subseteq$ $[1, n / 2]$, and $T \subseteq[1, m n / 2]$ with $T=d n R \cup d m S$ for some $d$ such that $\operatorname{gcd}(m n, d)=1$, then $C_{m n}(T) \cong C_{m}(R) \square C_{n}(S)$.

Note 5. From the factorization theorem, we get, in particular, when $\operatorname{gcd}(2 R(2 n+1), d)=1$ and $T=2 d R \cup\{2 n+1\}$, $C_{2(2 n+1)}(T) \cong P_{2} \square C_{2 n+1}(R)$ and when $\operatorname{gcd}(4 R(2 n+1), d)=1$ and $T=4 d R \cup\{2 n+1\}$ or $4 d R \cup\{2 n+1,2(2 n+1)\}, C_{4(2 n+1)}(T) \cong$ $C_{4} \square C_{2 n+1}(R)$ or $C_{4}(1,2) \square C_{2 n+1}(R)$, respectively.

Theorem 37. If $n \neq 4$ and $1 \in R$, then $C_{n}(R)$ is a prime circulant.

Proof. For $n=4, C_{n}=C_{4} \cong C_{2} \square C_{2}=P_{2} \square P_{2}$. Let $1 \in R$. For $n \neq 4$, if possible, assume that $C_{n}(R) \cong C_{n_{1}}(S) \square C_{n_{2}}(T)$ where $R, S$, and $T$ are sets of positive integers such that $R \subseteq[1, n / 2]$, $S \subseteq\left[1, n_{1} / 2\right], T \subseteq\left[1, n_{2} / 2\right], n_{1}, n_{2} \in \mathbb{N} \backslash\{1\}$, and $n=n_{1} n_{2}$. Then, using Theorems 26 and 27, there exist integer $d$ with $\operatorname{gcd}\left(n_{1} n_{2}, d\right)=1$ such that $C_{n_{1}}(S) \square C_{n_{2}}(T) \cong C_{n_{1} n_{2}}\left(d n_{2} S \cup\right.$ $\left.d n_{1} T\right)$. Now the length of periodic cycles of $C_{n_{1} n_{2}}\left(d n_{2} S \cup\right.$ $\left.d n_{1} T\right)$ is either $n_{1} n_{2} / \operatorname{gcd}\left(n_{1} n_{2}, d n_{2} s\right)=n_{1} / \operatorname{gcd}\left(n_{1}, s\right)$ or $n_{1} n_{2} / \operatorname{gcd}\left(n_{1} n_{2}, d n_{1} t\right)=n_{2} / \operatorname{gcd}\left(n_{2}, t\right), s \in S$ and $t \in T$. This implies that no periodic cycle of $C_{n_{1} n_{2}}\left(d n_{2} S \cup d n_{1} T\right)$ 
is of length $n=n_{1} n_{2}$, whereas $C_{n}(R)$ has periodic cycle (of period 1 and) of length $n$. This is a contradiction to $C_{n}(R) \cong$ $C_{n_{1} n_{2}}\left(d n_{2} S \cup d n_{1} T\right) \cong C_{n_{1}}(S) \square C_{n_{2}}(T)$ for some $d$ such that $\operatorname{gcd}\left(n_{1} n_{2}, d\right)=1$.

Remark 38. The converse of the above theorem is not true. That is, if $C_{n}(R)$ is a prime circulant graph, then $R$ need not contain 1 . For example, $C_{30}(3,4,5)$ is a prime circulant graph, using the factorization theorem, but it is not isomorphic to $C_{30}(1, r, s)$ for all possible values of $r$ and $s$.

Corollary 39. If $n \neq 4$ and if $R$ contains an integer relatively prime to $n$, then $C_{n}(R)$ is prime circulant.

Proof. Let $r \in R$ be relatively prime to $n$. Hence there exists $d \in \mathbb{Z}_{n}^{*}$ such that $d r \equiv 1(\bmod n)$. Clearly, $C_{n}(d R)$ is Adam's isomorphic to $C_{n}(R)$ and contains jump size 1 corresponding to $d r$ which is $\equiv 1(\bmod n)[22]$. Now the result follows from Theorem 37.

Corollary 40. If $n$ is a prime power other than 4 and $C_{n}(R)$ is connected, then $C_{n}(R)$ is prime circulant for all $R \neq \phi$.

Proof. Clearly $C_{p}(R)$ with $R=\left\{r_{1}, r_{2}, \ldots, r_{k}\right\} \neq \Phi$ is prime circulant for any prime number $p$. Let $n=p^{m} \neq 2^{2}$ where $p$ is a prime number and $m \in \mathbb{N}$. Given that $C_{p^{m}}(R)$ is connected which implies that $\operatorname{gcd}\left(p^{m}, r_{1}, r_{2}, \ldots, r_{k}\right)=1$. Since $p$ is prime, the above relation implies that there exists at least one $r_{i}$ such that $\operatorname{gcd}\left(p^{m}, r_{i}\right)=1,1 \leq i \leq k$. Now the result follows from Corollary 39.

Theorem 41 (fundamental theorem of circulant graphs). Every connected circulant graph is the unique product of prime circulant graphs (uniqueness up to isomorphism).

(It follows that if a connected circulant $C_{n}(T) \cong$ $C_{k_{1}}\left(R_{1}\right) \square C_{k_{2}}\left(R_{2}\right) \square \cdots \square C_{k_{m}}\left(R_{m}\right) \cong C_{l_{1}}\left(S_{1}\right) \square C_{l_{2}}\left(S_{2}\right) \square \cdots \square$ $C_{l_{h}}\left(S_{h}\right)$, where $C_{k_{i}}\left(R_{i}\right)$ and $C_{l_{j}}\left(S_{j}\right)$ are connected prime circulant graphs, then $m=h$ and there exists a permutation $\Pi$ on the symbols $1,2, \ldots, m$ such that $\Pi(i)=j, C_{l_{j}}\left(S_{j}\right) \cong$ $C_{k_{i}}\left(R_{i}\right), \operatorname{gcd}\left(k_{\alpha}, k_{\beta}\right)=1$ and $\operatorname{gcd}\left(l_{a}, l_{b}\right)=1, \alpha \neq \beta, a \neq b$ and $k_{\alpha}, k_{\beta}, l_{a}, l_{b} \neq 2$, each prime circulant and prime circulant of even order occur at the most once in the prime factorization except $C_{2}$ which may occur at the most twice, $n=k_{1} k_{2}, \ldots$, $k_{m}=l_{1} l_{2}, \ldots, l_{h}$, and $i, j, \alpha, \beta, a, b=1,2, \ldots, m$. Here we have if $A \square G \cong A \square H$, then $G \cong H$ for any graph $A$.)

Proof. Using the factorization theorem, we can write any circulant graph as a product of prime circulant graphs. The number of even order prime factors (circulant) of any given connected circulant is at the most two. Furthermore, in the case of having two even order prime factors, each prime factor is $C_{2}$ only. The uniqueness follows from Theorems 10, 15, 18, and 26-31, and Corollaries 17 and 25.

Note 6. In the above theorem we have if $A \square G \cong A \square H$, then $G \cong H$ for any graph $A$. But the converse need not be true always. That is if $G$ and $H$ are isomorphic connected simple graphs and $A$ is any connected simple graph, then $A \square G$ and $A \square H$ need not be isomorphic. For example, (i) $C_{16}(1,2,7) \square$
$C_{16}(1,2,7)$ is not isomorphic to $C_{16}(1,2,7) \square C_{16}(3,4,5)$, even though $C_{16}(1,2,7) \cong C_{16}(2,3,5)$. Another example is (ii) $C_{16}(1,2,7) \square C_{27}(1,3,8,10)$ is not isomorphic to $C_{16}(1,2,7) \square C_{27}(3,4,5,13)$, even though $C_{27}(1,3,8,10) \cong$ $C_{27}(3,4,5,13)$. The product graphs in example (i) are not circulant, whereas in example (ii) the product graphs are circulant graphs.

Remark 42. If $G$ is a connected graph such that $G \cong G_{1} \square G_{2} \square \cdots \square G_{k}$, then the diameter of $G, \operatorname{dia}(G)=$ $\sum_{i=1}^{k} \operatorname{dia}\left(G_{i}\right)[23]$.

Thus, we can find the diameter of any given circulant graph, provided that diameters of its prime circulant graphs are known. Also the above relation helps to generate (circulant) graphs of bigger diameters.

\section{Concluding Remarks}

(1) In prime factorization of connected circulants $C_{1}()=$ $K_{1}$ and $C_{2}=P_{2}$ act similar to 1 and 2 among the set of all natural numbers, respectively. Thus, $C_{1}()$ is a unit, like 1 in number theory.

(2) There exist two types of prime circulant graphs of order $n$, one with periodic cycle(s) of length $n$ and the other without periodic cycle of length $n$. See Theorem 37, Corollary 39, and Remark 38.

(3) The theory of factorization of circulants is similar to the theory of factorization of natural numbers and one of the very few well-known mathematical structures so vividly classified (expressed) in terms of prime factors. It can be applied in cryptography.

(4) POLY315.exe is a VB program developed by us to show visually how the transformation $\Phi_{m, n}$ acts on $C_{m} \square C_{n}$ for different values of $m$ and $n, m, n \in \mathbb{N}$.

(5) An interesting problem is, for a given integer $n$, finding the number of prime (composite) circulant graphs of order either equal to $n$ or less than or equal to $n$.

(6) One can develop theories similar to the theory of Cartesian product and factorization of circulant graphs to the other standard products of circulant graphs.

\section{Acknowledgments}

The author expresses his sincere thanks to Professor Lowell W. Beineke, Indiana-Purdu University, USA, Professor Brian Alspach, University of Newcastle, Australia, Professor M. I. Jinnah, University of Kerala, Thiruvananthapuram, India, and Professor V. Mohan, Thiyagarajar College of Engineering, Madurai, Tamil Nadu, India, for their valuable suggestions and guidance and Dr. A. Christopher, Dr. P. Wilson, and R. Satheesh of S. T. Hindu College, Nagercoil, India, for their assistance to develop the VB program. The author also expresses his gratitude to Lerroy Wilson Foundation (http://www.WillFoundation.co.in) for providing financial assistance to do this research work. Research is supported 
in part by Lerroy Wilson Foundation, Nagercoil, India (http://www.WillFoundation.co.in).

\section{References}

[1] R. Arratia, A. D. Barbour, and S. Tavare, "Random combinatorial structures and prime factorizations," Notices of the American Mathematical Society, vol. 44, no. 8, pp. 903-910, 1997.

[2] W. Imrich and H. Izbicki, "Associative products of graphs," Monatshefte für Mathematik, vol. 80, no. 4, pp. 277-281, 1975.

[3] I. Broere and J. H. Hattingh, "Products of circulant graphs," Quaestiones Mathematicae, vol. 13, no. 2, pp. 191-216, 1990.

[4] A. Adam, "Research problem 2-10," Journal of Combinatorial Theory, vol. 3, p. 393, 1967.

[5] B. Alspach and T. D. Parsons, "A construction for vertextransitive graphs," Canadian Journal of Mathematics, vol. 34, pp. 307-318, 1982.

[6] N. L. Biggs, Algebraic Graph Theory, Cambridge Uniersity Press, Cambridge, UK, 1974.

[7] F. T. Boesch and R. Tindell, "Circulant and their connectivities," Journal of Graph Theory, vol. 8, no. 4, pp. 487-499, 1984.

[8] F. Buckley and F. Harary, Distance in Graphs, Addison-Wesley, Reading, Mass, USA, 1991.

[9] P. J. Davis, Circulant Matrices, Wiley, New York, NY, USA, 1979.

[10] I. Kra and S. R. Simanca, "On circulant matrices," Notices of the American Mathematical Society, vol. 59, no. 3, pp. 368-377, 2012.

[11] R. S. Sanders, "Products of circulant graphs are metacirculant," Journal of Combinatorial Theory B, vol. 85, no. 2, pp. 197-206, 2002.

[12] R. S. Sanders and J. C. George, "When is the product of two circulant graphs circulant?” http://arxiv.org/abs/math/9907119. In press.

[13] V. Vilfred, "Circulant graph isomorphism-type-II," in Proceedings of the International Conference on Combinatorics, Statistics, Pattern Recognition and Related Areas, Abstract P75, Mysore University, Karnataka, India, December 1998.

[14] V. Vilfred, "A theory of Cartesian product and factorization of circulant graphs," in Proceedings of the International Conference on Emerging Trends in Mathematics and Computer Applications, pp. 54-57, Mepco Schlenk Engineering College, Sivakasi, India, December 2010.

[15] V. Vilfred, "Circulant graphs-isomorphism and factorization," in Proceedings of the National Seminar on Algebra and Discrete Mathematics, pp. 52-58, Kerala University, Thiruvananthapuram, India, October 2002.

[16] V. Vilfred, "Circulant graphs-self-complementary, isomorphism and factorization using VB programs," in Proceedings of the International Conference on Mathematics and Computer Science, pp. 125-128, Loyola College, Chennai, India, March 2007.

[17] V. Vilfred, "New Abelian groups from isomorphism of circulant graphs," in Proceedings of the of International Conference on Applied Mathematics and Theoretical Computer Science, pp. 1316, St. Xaviers Catholic Engineering College, Tamil Nadu, India, 2013.

[18] V. Vilfred, E-labelled graphs and circulant graphs [Ph.D. thesis], University of Kerala, Thiruvananthapuram, India, 1994.

[19] X. Fang and M. Xu, "On isomorphism of cayley graphs of small valency," Algebra Colloquium, vol. 1, no. 1, pp. 67-76, 1994.

[20] G. Sabidussi, "Graph multiplication," Mathematische Zeitschrift, vol. 72, no. 1, pp. 446-457, 1959.
[21] F. Harary, Graph Theory, Addison-Wesley, Reading, Mass, USA, 1969.

[22] T. M. Apostol, Introduction to Analytic Number Theory (Ex.25, Chapter 1), Springer, Berlin, Germany, 1989.

[23] J. Nieminen, "The center problem in the product of graphs," in Recent Studies in Graph Theory, V. R. Kully, Ed., Vishwa International, Gulbarga, India, 1989. 


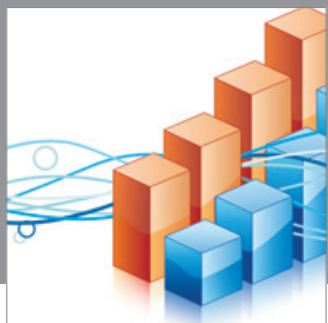

Advances in

Operations Research

mansans

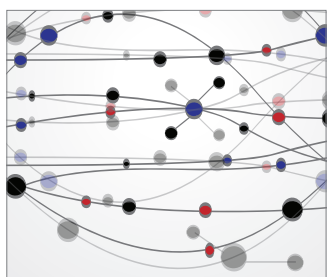

The Scientific World Journal
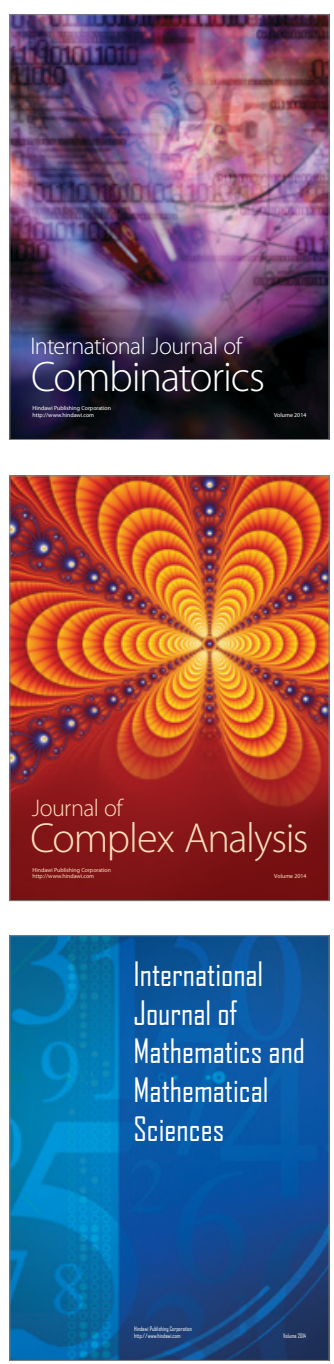
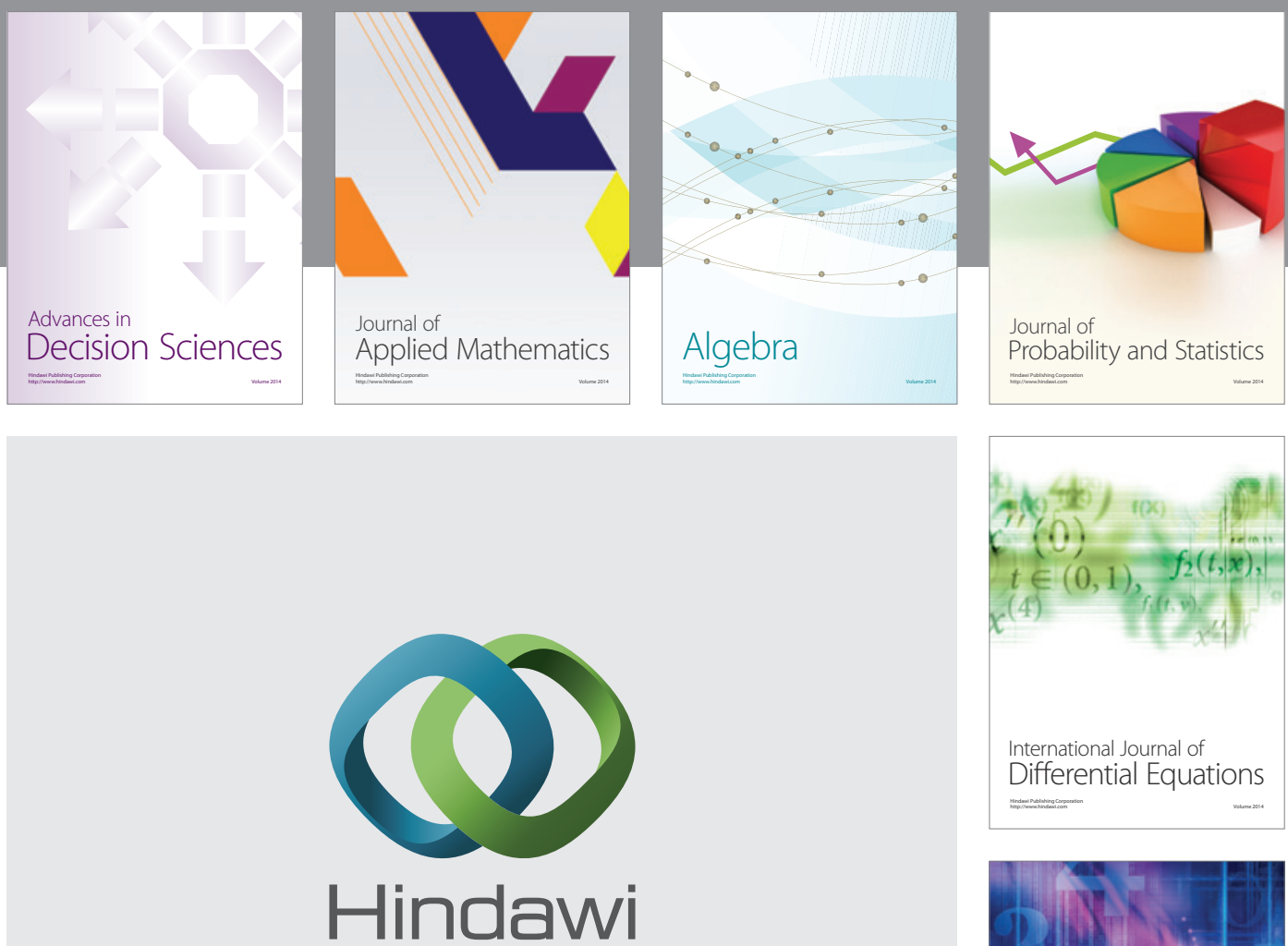

Submit your manuscripts at http://www.hindawi.com
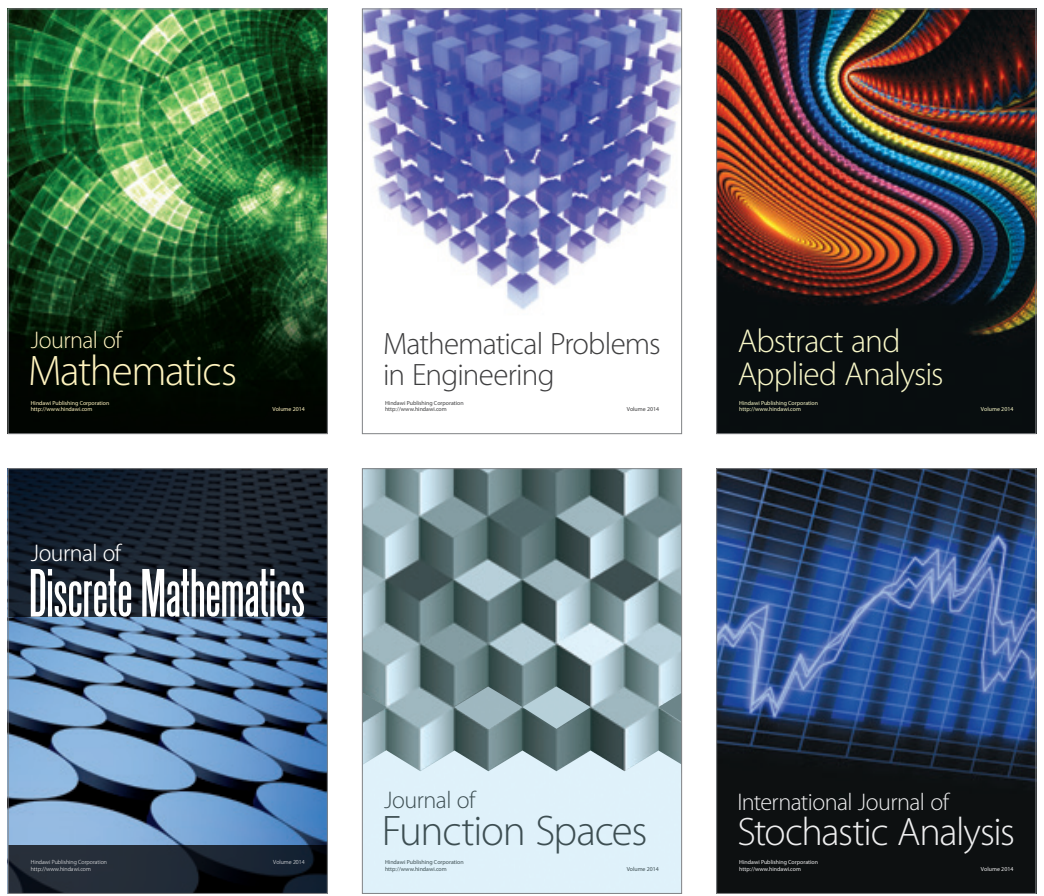

Journal of

Function Spaces

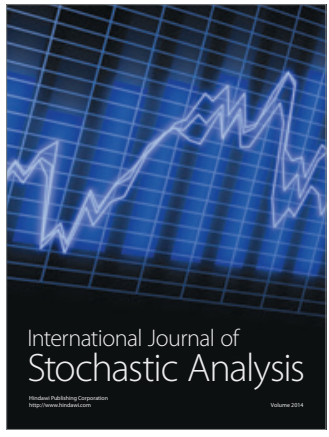

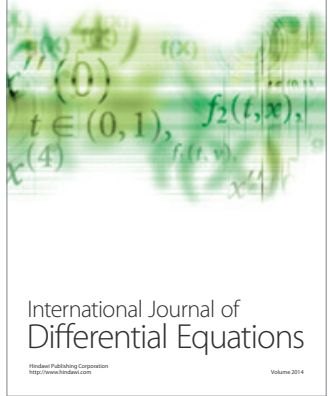
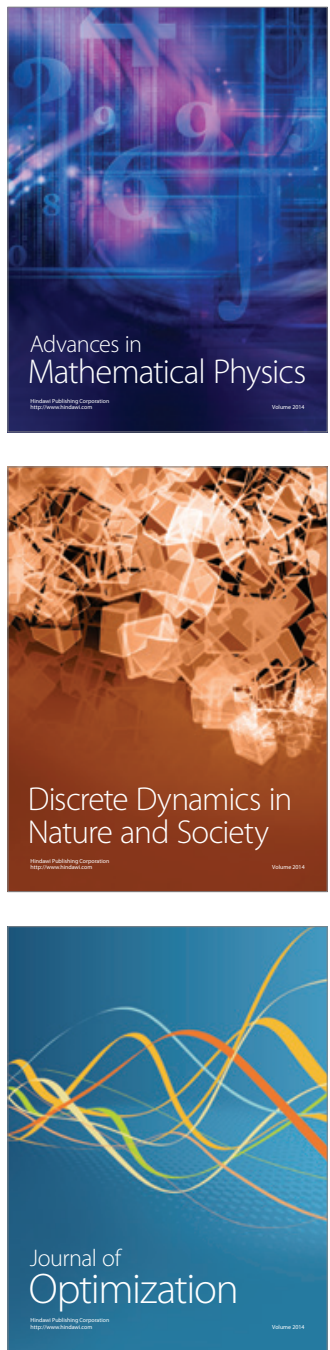